\title{
Editorial
}

Respiration

\section{Lessons from a Valve Trial}

\author{
Tudor P. Toma \\ Department of Respiratory Medicine, University Hospital Lewisham, London, UK
}

Bronchoscopic placement of valves for lung volume reduction is one of the many procedures now being developed to replace surgical lung volume reduction. There are at least 5 different methods of bronchoscopic lung volume reduction (BLVR), involving valves, spigots, coils, steam and biological glue [1]. All are in different stages of development and none is in current clinical practice.

In this issue of Respiration, Sterman et al. [2] report results from a pilot multicenter trial with Spiration $\mathrm{IBV}^{\circledR}$ unidirectional valves. The valves were used for bilateral treatment of severe heterogeneous upper-lobe predominant emphysema. The trial results suggest that bronchial valve treatment is safe, can improve quality of life in the majority of patients and has multiple mechanisms of action.

Most patients with severe chronic obstructive pulmonary disease are managed in the community or in a local hospital. There is thus a large number of respiratory physicians around the world that are primarily interested to hear when, and if, any BLVR treatments are going to be available in their local hospital. So how does the trial of Sterman et al. [2] contribute to the advancement of BLVR to routine clinical practice?

Safety, and in particular the risk of post-obstructive pneumonias, was the main initial concern with BLVR. Sterman et al. [2] confirm what has been known from earlier work, that a bronchial occlusion with a one-way valve is safe overall and has little influence on the risk of pneumonias [3].

\section{KARGER}

Fax +41613061234 E-Mail karger@karger.ch www.karger.com (c) 2010 S. Karger AG, Basel

0025-7931/10/0793-0191\$26.00/0

Accessible online at:

www.karger.com/res
There are several theories to explain why post-obstructive pneumonia is not frequent with endobronchial valves. One relies on the postulation that the valve blocks mainly the inspiratory flow, while allowing some expiratory flow and mucus drainage. Thus, lung segments distal to the valve are exposed to fewer particles and microorganisms, and those that do get through can still be cleared. However, when a tumour or a true foreign body is present, there is residual inspiratory flow and particulate entry in the distal segments, the expiratory clearance is impaired, and local infections are more frequent. If this is true, then valve design and correct valve placement are essential for procedure's safety. Better understanding of these observations is important for clinical progression of BLVR. However, much more research is required if we are to understand the factors that hinder or foster pneumonias with the use of valves.

An unexpected complication observed with valves and confirmed by Sterman et al. [2] is the increased risk of pneumothorax after the procedure. Pneumothoraces were observed in trials performed with other valves as well, and therefore they are unlikely to be a complication specific to the Spriation device [3]. Interestingly, when Sterman et al. [2] modified their targeting strategy to exclude treatment of the lingular segments, the post-procedure pneumothorax rate diminished. This observation suggests that the treatment strategy can improve the safety of BLVR.
Tudor P. Toma

Department of Respiratory Medicine, Lewisham University Hospital

Lewisham High Street

London SE13 6LA (UK)

Tel. +44 208333 3000, ext.6714, Fax +44 208333 3092, E-Mail ttoma@ doctors.org.uk 
In terms of efficacy, in our experience, confirmed by the yet unpublished VENT trial, patients who benefited most from BLVR performed with a similar valve (Emphasys) were those who had complete lobar occlusion and showed maximum volume reduction $[3,4]$. This simply means that, although there might be different mechanisms of action involved, the benefit becomes acceptable when volume reduction does occur.

Meanwhile I cannot agree more that there are other mechanisms of action involved. We struggled with our initial studies to explain why some patients with no atelectactic change after the procedure were still reporting subjective improvements. As shown by Sterman et al. [2], clinical benefits can occur through different mechanisms of action, but these seems to be small and variable. However, what is 'small' for some is probably 'huge' for a severely dyspnoeic COPD patient, and therefore these newly observed mechanisms deserve further studies.

Moreover, the efficacy results observed by Sterman et al. [2] illustrate how important is to gain enough experience with a new device, before moving into more com- plex, and expensive, randomised trials. BLVR development has been heavily influenced by regulatory requirements derived from surgical lung volume reduction work and from the NETT trial. As a consequence, every start up company working on a BLVR device was set by regulators to do pivotal trials similar to NETT. Those companies who went too early into large randomised trials quickly ended up bankrupt. Trials such as VENT were costly and failed to show clinically significant results. This is probably not because BLVR is ineffective, but because the choice of primary outcomes, patient selection and valve insertion strategies were not fully understood at the time of the trial's design.

Respiratory physicians should not be disappointed that the trial of Sterman et al. [2] is not going bring BLVR immediately into routine practice. They should be hopeful: BLVR is now better understood, and because is simple and is safe, trials may extend into the local hospitals where most patients with debilitating hyperinflation are treated.

\section{References}

1 Ingenito EP, Wood DE, Utz JP: Bronchoscopic lung volume reduction in severe emphysema. Proc Am Thorac Soc 2008;5:454460.

-2 Sterman DH, Mehta AC, Wood DE, et al: A multicenter pilot study of a bronchial valve for the treatment of severe emphysema. Respiration 2010;79:222-233.
3 Wan IYP, Toma TP, Geddes DM, et al: Bronchoscopic lung volume reduction for endstage emphysema. Chest 2006;129:518-526.

-4 Hopkinson NS, Toma TP, Hansell DM, Goldstraw P, Moxham J, Geddes DM, et al: Effect of bronchoscopic lung volume reduction on dynamic hyperinflation and exercise in emphysema. Am J Respir Crit Care Med $2005 ; 171: 453-460$. 